\title{
Permanent cardiac pacing in elderly patients with recurrent falls, dizziness and syncope, and a hypersensitive cardioinhibitory reflex
}

\author{
JG Crilley, B Herd, CS Khurana, CA Appleby, MA de Belder, A Davies, JA Hall
}

Department of Cardiology, Cardiothoracic Division, South Cleveland Hospital, Middlesbrough, UK JG Crilley,

CS Khurana,

CA Appleby,

MA de Belder,

A Davies,

JA Hall

Department of Medicine and Elderly Care, North Tees General Hospital, Hardwick, Stockton on Tees, Cleveland, UK

B Herd

Correspondence to Dr JA Hall, Department of Cardiology, South Cleveland Hospital, Marton Road, Middlesbrough TS4 3BW, UK

Accepted 30 October 1996

\begin{abstract}
Summary
The study was designed to assess the outcome of treatment with permanent dual-chamber pacing of elderly patients with falls, dizziness and syncope associated with the demonstration of a hypersensitive cardioinhibitory reflex. Questionnaires were sent to patients (and their general practitioners) who had been referred to a regional pacing centre with recurrent falls, dizziness or syncope diagnosed as likely to be secondary to cardioinhibitory carotid sinus syndrome or predominantly cardioinhibitory vasovagal syndrome. After pacemaker insertion, $84 \%$ of patients had no further syncope over a mean follow-up period of 10 (range 1.5 to 30 ) months. Minor symptoms persisted in only $40 \%$ of all patients. Symptoms were unchanged in $22 \%$. It was concluded that permanent dual-chamber pacing is an effective treatment for elderly patients with recurrent falls, dizziness and syncope in whom a hypersensitive cardioinhibitory reflex is found. Good results were obtained in this group with a simple diagnostic work-up.
\end{abstract}

Keywords: cardiac pacing, elderly, syncope, hypersensitive cardioinhibitory reflex

Falls, dizziness and syncope are common problems in the elderly and may result in significant injury. ${ }^{1}$ Ascribing a single diagnosis for the cause of symptoms in such patients can be difficult due to coexistent chronic illnesses, multiple drug therapy and overlap of aetiologies. In an attempt to establish a specific cause for symptoms, patients may be investigated with carotid sinus massage (CSM) and/or head-up tilt test (HUTT), both of which may reproduce the dizziness or syncope which is associated with bradycardia (cardioinhibitory response), hypotension (vasodepressor response) or both (mixed response). ${ }^{2}$ Permanent cardiac pacing has been demonstrated to be an effective treatment for patients with cardioinhibitory responses to CSM or HUTT and recurrent syncope and pre-syncope. ${ }^{3-6}$

However, patients assessed in clinical trials may not be representative of those seen in clinical practice. Trial patients have often been highly selected and only included if they have had reproducible symptoms with repeated
CSM or HUTT. Often, patients have been pre-assessed with temporary pacing systems to establish abolition of symptoms by pacing prior to permanent pacemaker insertion. A wide variety of possible confounding medical illnesses have usually been excluded by extensive investigations.

It is not always practical to investigate patients so intensively in routine practice. We have adopted a more pragmatic approach to the management of syncope, pre-syncope and falls in the elderly. We implant dual-chamber pacemakers in all symptomatic patients with a cardioinhibitory response to testing in whom this is likely to be a major contributing factor. The aim of this review was to determine the outcome of this approach.

\section{Methods and subjects}

Patients with recurrent falls, dizziness and syncope were usually assessed by a geriatrician (BH, 65\%). Patients in whom a detailed history, clinical examination and Holter recording failed to reveal another clear cause of syncope proceeded to CSM and in some cases HUTT. A positive cardioinhibitory response was defined as a ventricular pause of $>3$ seconds after 5 seconds massage of either carotid sinus in the supine or erect positions.

Forty-two patients who had recurrent falls, dizziness or syncope and had cardioinhibitory responses to CSM and/or HUTT were identified between April 1993 and January 1996. These patients were implanted with dualchamber pacemakers programmed to DDI with rate hysteresis $(50-90$ beats $/ \mathrm{min})$ using Physios $0.1^{\kappa \star}$, Reflex ${ }^{\mathrm{k} \star \star}$ or Paragon III ${ }^{\mathrm{k} \star \star \star}$ generators.

In order to assess the patient's response to pacemaker insertion a questionnaire was sent to the general practitioner and to the patient. We requested information from the general practitioner about the presence or absence of post-implantation falls, dizzy spells and blackouts. A similar questionnaire was sent to the

* Biotronik GmbH \& Co, Woermannkehre 1, D-12359, Berlin, Germany

$\star \star$ Telectronics Pacing Systems, 7400 South Tucson Way, Englewood, CO 80112, USA

$\star \star \star$ Pacesetter, St Jude Medical Company, S-171 95 SOLNA, Sweden 
patients. This asked them whether they had had any falls, dizzy spells or blackouts before implantation of the pacemaker and whether they had experienced similar symptoms afterwards. If so, we asked whether the frequency of symptoms had altered since the pacemaker was implanted.

\section{Results}

The 42 patients comprised 18 men and 24 women. Their average age at implantation was 79 (range 58-91) years. The length of followup was an average of $10(1.5-30)$ months. Survival data were available for all patients and follow-up symptomatology data available for 39 patients including all those still alive. The questionnaire was returned by 33 of the 34 patients who were alive at the time of followup. In addition, two patient's relatives returned the questionnaire after their relative had died. Information about four of the remaining patients was obtained from the general practitioner.

Thirty-seven patients were paced due to demonstration of a bradycardic response to CSM on one occasion. Three patients had a positive response to CSM and a bradycardic response on tilt-testing. These 40 patients were classified as having carotid sinus syndrome. Two patients had a positive HUTT (bradycardic and vasodepressor response) and a negative response to CSM. These patients were classified as having malignant vasovagal syndrome.

\section{CAROTID SINUS SYNDROME}

Of the patients with carotid sinus syndrome (for whom there were both pre- and postimplant data), 30 patients ( $81 \%$ ) had falls, 28 patients $(76 \%)$ dizziness, and $24(65 \%)$ syncope prior to pacemaker insertion. Falls or syncope were present in 34 patients $(92 \%)$ and dizziness only in three patients $(8 \%)$. Four patients $(11 \%)$ had sustained significant injury prior to pacing; these included three fractures and a head injury without skull fracture.

Symptoms improved markedly after implantation of the pacemaker. Only six patients (16\%) reported syncope, 16 patients (43\%) dizziness and 11 patients $(30 \%)$ falls $(\mathrm{p}<0.0001, \mathrm{p}<0.01$, and $\mathrm{p}<0.0001$ for each symptom, respectively (figure 1)). Falls or syncope were present in 14 patients (38\% $(\mathrm{p}<0.0001))$. Of the 24 patients who originally reported syncope, 20 patients $(83 \%)$ reported no further syncope after pacemaker insertion.

The overall response to pacemaker insertion is shown in figure 2 . One patient who initially reported persistent symptoms had relief of their symptoms with increasing the rate hysteresis to $60-100$ beats/min.

Eight patients $(20 \%)$ died within the followup period; post-implantation symptomatology data were available in five of these and three died before follow-up. Two patients who later died had been completely relieved of their symptoms. The causes of death were malignancy (2), cerebrovascular disease (3), ischaemic heart disease (2) and ruptured abdominal aortic aneurysm (1). These patients died an

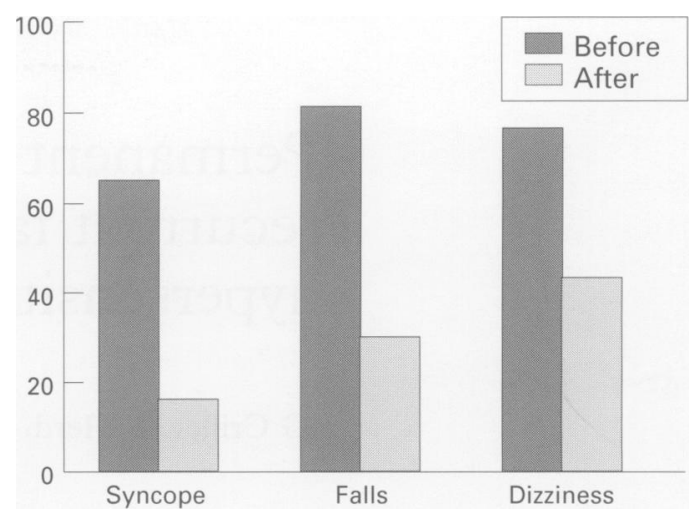

Figure 1 Percentage of patients with carotid sinus syndrome having symptoms before and after pacemaker insertion

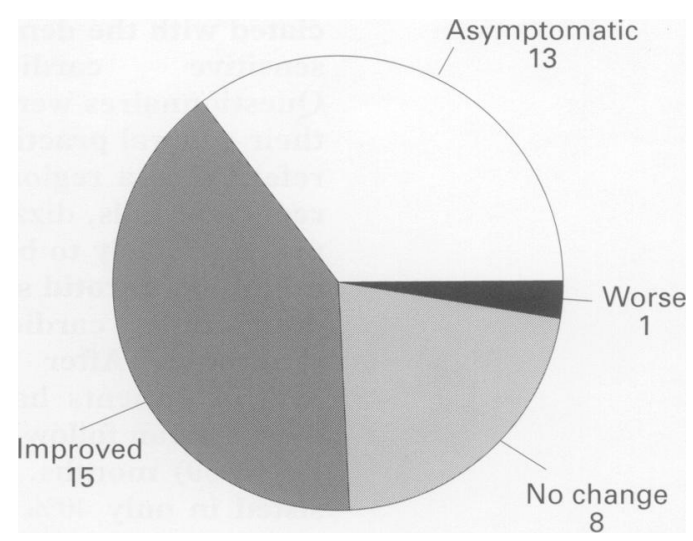

Figure 2 Patients with carotid sinus syndrome: response to cardiac pacing

average of seven months after implantation of the pacemaker.

MALIGNANT VASOVAGAL SYNDROME

The two patients with malignant vasovagal syndrome both had no further syncope. One patient reported persistent minor symptoms and the other reported no further symptoms.

\section{COMPLICATIONS}

There was one significant complication related to pacemaker insertion. One patient had an early.erosion of the generator; the system was removed and a second system inserted on the contralateral side without further complication.

\section{Discussion}

Fall, dizziness and syncope are common symptoms in the elderly. One study of institutionalised patients found an annual incidence of syncope of $6 \%$ with a recurrence rate of $30 \% .^{7}$ Another found that $36 \%$ of elderly patients have at least one fall per year. ${ }^{8}$ Falls, dizziness or syncope account for approximately $20 \%$ of UK acute geriatric hospital admissions. ${ }^{9}$ One-third of elderly patients who fall due to syncope have retrograde amnesia for the associated syncopal attack which is described by a witness. ${ }^{2}$ In addition, background difficulties with short-term memory may make interpretation of a questionnaire unreliable. 
We therefore elected to use a simple questionnaire which asked patients about the presence or absence of symptoms and a brief comment about the effect of the pacemaker rather than to ask them to assess in detail the frequency of their symptoms as has been used in some other studies. ${ }^{4,10}$

Recurrent falls and syncope place a heavy burden on carers and result in loss of confidence for the patient. Systematic evaluation of a patient with recurrent falls or syncope is important since establishing a diagnosis and instituting effective therapy may not only result in a much improved quality of life for the patient and family but may also avoid injury and maintain mobility.

There are no universal protocols for the investigation of patients with falls, dizziness and syncope. Patients may present to a general practitioner, casualty department, orthopaedic surgeon, neurologist, cardiologist or geriatrician. Investigations may include electroencephalogram, ${ }^{11}$ ambulatory electrocardiogram, ${ }^{12}$, gait analysis, ${ }^{13}$ muscle strength testing, ${ }^{14}$ invasive electrophysiological testing ${ }^{12}$ and risk stratification based on miltivariate analysis. ${ }^{15,16}$

In some units it has become routine practice to perform CSM and/or HUTT on any elderly patient with a fall or syncopal attack whose routine investigations including Holter recording are unrewarding. However, having demonstrated an abnormal response to these investigations, the decision regarding the appropriate interventions is not widely agreed. It remains unclear which patients should be offered treatment with pacing and what benefits might be anticipated.

The significance of a positive response to CSM has been questioned since the frequency of an abnormal response has been estimated to be at least $10 \%$ in asymptomatic patients and even higher in those with coronary artery disease or hypertension. Brown et al found a positive response to CSM in $32 \%$ of patients undergoing elective coronary angiography ${ }^{17}$ although none of the patients had associated symptoms. In another study of patients who had a positive response to CSM, $90 \%$ of patients who were asymptomatic remained so, whilst syncope recurred in $50 \%$ of the patients who were symptomatic. ${ }^{18}$ It has been suggested that a positive response to CSM is only a marker of neurally mediated syncope and hence a decision regarding permanent pacing should be taken in light of the presence or absence of associated symptoms.

There appears to be wide variation in the frequency of diagnosis of carotid sinus syndrome varying from $0.5 \%$ of patients evaluated for unexplained syncope ${ }^{19}$ to $45 \%$ of patients referred to a tertiary syncope clinic. ${ }^{10}$ Our approach is to diagnose carotid sinus syndrome in patients with recurrent falls, dizziness and syncope if CSM is positive. We proceed to permanent pacemaker implantation without the hazards of pre-implant temporary pacing or the unreliability of repeat testing.

Several studies have demonstrated a benefit of pacing in carotid sinus syndrome although few have included more than 30 cases (table).
Table Comparison of the results of this study with published reports

\begin{tabular}{llll}
\hline Reference & $n$ & $\begin{array}{l}\text { Persistent severe } \\
\text { symptoms (\%) }\end{array}$ & $\begin{array}{l}\text { Persistent minor } \\
\text { symptoms (\%) }\end{array}$ \\
\hline 3 & 70 & 17 & not reported \\
5 & 60 & 13 & 44 \\
4 & 32 & 16 & 73 \\
present & 40 & 16 & 40 \\
\hline
\end{tabular}

Morley et al analysed 70 patients who had been treated for carotid sinus syndrome with permanent pacing and had been followed over four years. ${ }^{3}$ Eighty-three per cent $(58 / 70)$ had relief of symptoms. More than $90 \%$ of patients received VVI generators. Persistent symptoms were attributed to 'pacemaker syndrome' or vasodepressor symptoms which were not treated by adequate bradycardia control. Two of these patients had their symptoms ameliorated with conversion to a dual-chamber system. Of note is that all patients were pre-assessed with temporary pacing systems to establish abolition of symptoms prior to permanent pacemaker insertion.

Brignole et al randomised 60 patients with severe carotid sinus syndrome to either pacing or non-pacing groups. ${ }^{4}$ All patients had reproducible abnormal responses to CSM on two occasions. Thirty-two patients received permanent pacemakers (though only 14 of these were dual-chamber units). They found that patients randomised to the pacing group had significantly fewer symptoms than patients in the non-pacing group. There was no significant difference in outcome between those who received VVI units and those who received DDD units, although the numbers of patients were small. Again, patients were assessed prior to permanent pacemaker insertion by temporary pacing during CSM to evaluate the likely success of VVI pacing versus DDD pacing. In spite of this prior assessment, $16 \%$ of patients had persistent syncope and $73 \%$ further minor symptoms during four years of follow-up. In a previous non-randomised study of 60 patients, Brignol et $a \bar{l}$ found that $13 \%$ of patients had persistent severe symptoms and $44 \%$ minor symptoms during 32 months of follow-up.

Although it appears that there are some patients in whom VVI pacing is adequate, it is generally agreed that dual-chamber pacing is the mode of choice in carotid sinus syndrome but there are few published data concerning the benefits of DDI with rate hysteresis over conventional DDD pacing. DDI mode reduces the incidence of pacemaker-mediated tachycardia, which may occur in patients whose AV node function is intact if they are implanted with DDD pacing systems. Rate hysteresis allows a rate of pacing higher than the normal resting heart rate during activation of the cardioinhibitory reflex to improve the resolution of any associated peripheral vasodilation whilst affording a lower back-up rate to prevent unnecessary pacing during nocturnal physiological bradycardia. Peripheral vadodilatation has been found to be a significant contributing factor in patients who continue to have 
syncope despite pacing, ${ }^{3}$ although this may be overcome, as we achieved in one patient, by increasing the hysteresis rate. Other options include combining a permanent pacemaker with pharmacological therapy such as betablockers in patients who continue to be symptomatic. However, treatment of the peripheral vasodilatation component of patients' symptoms has not been satisfactory.

The significance of an abnormal HUTT in elderly patients is also questioned with there being wide variation in the reports of its frequency. McIntosh et al identified malignant vasovagal syndrome as a cause of recurrent syncope in $11 \%$ of patients referred to their syncope clinic. ${ }^{10}$ This diagnosis is thought to be more common in young adults but its prevalence in the elderly may simply be underrecognised due to cross-over with other forms of cardioinhibitory and vasodepressor syncope. Facilities for HUTT are less readily available and thus patients may be less often investigated in this way.

Pacing in malignant vasovagal syndrome has met variable acceptance. This is because it is widely accepted that the vasodepressor response which accompanies the bradycardia is untreated by pacing and that peripheral vasodilatation is often the major cause of patients' symptoms. However, Fitzpatrick and Sutton achieved good results in a study of 10 patients in whom they assessed patients using temporary dual-chamber pacing during HUTT. ${ }^{6}$ They found that $85 \%$ of subjects in whom the tilt response had been predominantly cardioinhibitory had relief of their symptoms. The mean age of their patients was 67 years. Although the

1 Nevitt MC, Cummings SR, Hudes ES. Risk factors for injurious falls: a prospective study. $\mathcal{F}$ Gerontol 1991; 46: $164-70$.

2 McIntosh S, Lawson J, Kenny RA. Clinical characteristics of vasodepressor, cardioinhibitory and mixed carotid sinus syndrome in the elderly. Am f Med 1993; 95: 203-8.

3 Morely CA, Perrins EJ, Grant P, Chan SL, McBrien DJ, Sutton R. Carotid sinus syncope treated by pacing; analysis Sutton $R$. Carotid sinus syncope treated by pacing; analysis
of persistent symptoms and role of atrioventricular sequenof persistent symptoms and role of atriovent

4 Brignole M, Menozzi C, Lolli G, Bottoni N, Gagglioli G. Long-term outcome of paced and nonpaced patients with Long-term outcome of paced and nonpaced patients with
severe carotid sinus syndrome. Am $\mathcal{F}$ Cardiol 1992; 69:
$1039-43$.

5 Brignole M, Menozzi C, Lolli G, Oddone D, Gianfranchi L, Bertulla A. Validation of a method of pacing mode in carotid sinus syndrome with or without sinud bradycardia. PACE 1991; 14: 196-203.

6 Fitzpatrick A, Theodorakis G, Ahmed R, Williams T, Sutton R. Dual chamber pacing aborts vasovagal syncope induced by head-up $60^{\circ}$ tilt. PACE 1991; 14: 13-9.

$7 \mathrm{Lipsitz} \mathrm{LA,} \mathrm{Wei} \mathrm{JY,} \mathrm{Rowe} \mathrm{JW.} \mathrm{Syncope} \mathrm{in} \mathrm{an} \mathrm{elderly,}$ institutionalized population: prevalence incidence and institutionalized population: prevalence

8 Hale WA, Delaney MJ, McGaghie WC. Characteristics and

8 Hale WA, Delaney MJ, McGaghie WC. Characteristics and
predictors of falls in elderly patients. 7 Fam Pract 1992; 34: predictors of falls in elderly patients. F Fam Pract 1992; 34:
$577-81$. $577-81$

9 Naylor R, Rosin AJ. Falling as a cause of admission to a geriatric unit. Practitioner 1970; 205: 327-30.

10 McIntosh S, Da Costa D, Kenny RA. Outcome of an integrated approach to the investigation of dizziness, falls, and syncope in elderly patients referred to a 'syncope' clinic. Age Ageing 1993; 22: 53-8.

\section{Learning points}

- a hypersensitive cardioinhibitory reflex is a common cause of syncope in older people

- patients who might benefit from permanent cardiac pacing can be identified by carotid sinus massage and head-up tilt test without the need for more invasive investigations

- most patients can expect relief of syncope although half will continue to have minor dizziness

two patients we paced for this reason responded well, the number is too small to comment on its efficacy.

In summary, the effective management of falls, dizziness and presyncope in the elderly can be a considerable challenge. There are no universally accepted protocols for investigation, diagnosis and treatment. Using a simple strategy of evaluating patients with CSM followed by HUTT (if negative) and pacing those patients with cardioinhibitory responses to CSM or HUTT, we have found that $83 \%$ have relief of syncope. We also found that all symptoms can be abolished in $35 \%$ of patients and a further $40 \%$ symptomatically improved with implantation of dual-chamber pacemakers programmed to DDI with rate hysteresis. We have chosen this straightforward approach to the selection of patients for pacing and have found a response rate in our general elderly population which is similar to that in more highly selected and intensively investigated groups.

11 Davis TL, Freeman FR. EEG should not be routine in the evaluation of syncope adults. Arch Intern Med 1990; 150: 2027-9.

12 Kapoor WN. Syncope in older persons. $7 \mathrm{Am}$ Geriatr Soc 1994; 42: 426-36.

13 Sudarsky L. Geriatrics: gait disorders in the elderly. $N$ Engl f Med 1990; 322: $1441-6$.

14 Lord SR, Ward JA, Williams P, Anstey KJ. Physiological factors associated with falls in older community-dwelling factors associated with falls in older comm

15 Tinetti ME, Baker DI, McAvay G, et al. A multifactorial intervention to reduce the risk of falling among elderly people living in the community. $N$ Engl f Med 1994; 331: $821-7$.

16 Rubenstein LZ, Robbins AS, Josephson KR, Schulman BL, Osterweil D. The value of assessing falls in an elderly population - a randomized clinical trial. Ann Intern Med 1990; 113: 308-16.

17 Brown KA, Maloney JA, Smith HC, Hartzler GO, Ilstrup GM. Carotid sinus reflex in patients undergoing coronary angiography: relationship of degree and location of coronary artery disease to response to carotid sinus massage. artery disease to response to
Circulation 1980; 62: $697-703$. 18 Blanc J, Boshat J, Penther P. Hypersensibilité sinocarotidienne. Evolution à moyen terme en fonction du traitem

19 Kapoor WN. Diagnostic evaluation of syncope. $\mathrm{Am} \mathcal{F} \mathrm{Med}$ 1991; 90: $91-106$ 